\title{
CV-SIFT Algorithm for Image Detection
}

\author{
Yuyin Cui,Yunting Lei \\ Key Laboratory of Information Network Security, Ministry of Public Security, \\ People's Republic of China (The Third Research Institute of Ministry of Public Security) \\ Shanghai, China \\ cuiyuyin@stars.org.cn, leiyunting@stars.org.cn
}

\begin{abstract}
With the development of technology, digital image tampering technology is becoming mature. It is difficult to directly determine from the naked eye whether a digital image has been modified. Furthermore, many digital watermarking in digital images cannot be identified, since the importance of it is neglect .In view of the wide use of digital image, such as evidence in court, a series of algorithms are designed to detect whether the digital image has been tampered.
\end{abstract}

Keywords- digital image, tampering algorithms, digital watermarking

\section{INTRODUCTION}

Copy-Paste tampering is a tamper techniques for the local region in digital image [1], as the most common image tamper method that it is a interception copy in a digital image, and paste the copied region to other position in the same image, and then post-process with retouching, background brightness, etc, thus hiding some important information in the image. General, an image with copy-paste tamper has two or more similar regions.

\section{ORIGINAL SIFT ALGORITHM ANALYSIS}

\section{A. SIFT algorithm analysis}

SIFT (Scale Invariant Feature Transform) algorithm [2], namely Scale Invariant Feature Transform algorithm, first proposed by David G. Lowe in 1999 and raised in 2004 to achieve a scale space-based for image scaling, rotation and affine transformation remain invariant characterization of digital image operator. Since its expansion algorithm the SIFT algorithm and is its expansion algorithm described in the same algorithm has the most robust stability, there are widely used of Copy-Paste tampering detection on digital image.

SIFT feature is the local feature of digital images, it remain invariance of scaling, rotation ,and also keep a certain degree of stability with change of brightness, the viewing changed, affine transformation and noise.

\section{B. SURF algorithm analysis}

SURF (Speeded up Robust Feature) is a highly robust gravid arum feature point detection algorithm [3], by Herbert Bay proposed in 2006. The algorithm can be used in computer vision field of object recognition. According to the authors, the algorithm is more efficient than the SIFT algorithm with strong robustness, the algorithm using integral image, Haar wavelet transform and approximate Hessian matrix operations to improve time efficiency, using Haar wavelet transform to increase the robustness.

\section{SIFT-BASED COLOR IMAGE EDGE DETECTION METHOD}

\section{A. Design ideas}

Though the SIFT Algorithm and SURF algorithm have an excellent performance, but this type algorithm is mainly use for the gray-scale image processing, but ignored the color information. On this color image dominated world, those algorithms have been limited [4].

This paper proposes an enhancement SIFT Algorithm based on color edge detection method for color digital images (CV-SIFT algorithm). At first, color images should converted to RGB color space, and then take edge extraction on image with the Vector-Order color edge detection operator, which in the RGB color space. After that, use GLCM to extract edge features in different color space, and process the feature points with SIFT algorithm which extracted for feature points sets produce and feature match, and then each feature point sets separate and recursive partition until to find all the matching point pairs. The process shown in Fig.1.

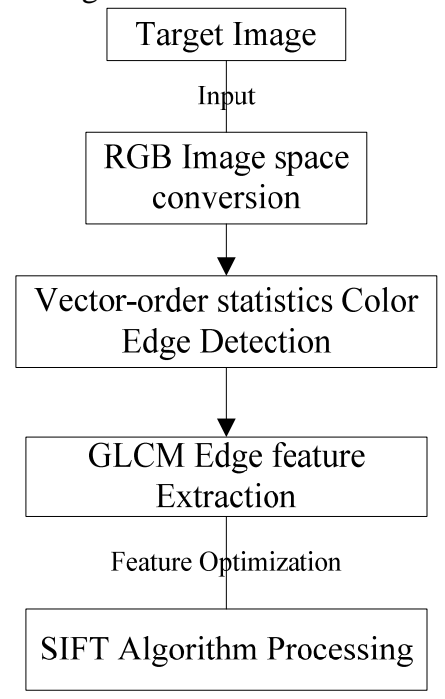

Figure 1. SIFT Detection Method Based on Color image edge 


\section{B. Analysis of key technologies}

1) Vector-order color edge extraction. Vector-order color edge has extracted by the vector space method, scilicet set one pixel as a different color components of the vector (e.g.: $[R, G, B]$ ). When the color edge has been extracted, the operation target is no longer a gray value but a vector. The $X=\left(x_{1}, x_{2}, \ldots, x_{p}\right)$ as a vector containing $P$ components and the $x_{l}, l=1,2, \ldots, p$ is random variable $X_{i}$ as $X$ 's the observed values. The sum of Distance for ${ }^{X_{i}}$ vector and the other vector is defined as:

$$
d_{i}=\sum_{k=1}^{n}\left\|X_{i}-X_{k}\right\|
$$

Such a sequence $d_{(1)}<d_{(2)}<\ldots<d_{(n)}$ can be obtained, According to this arrangement to obtain a corresponding arrangement $X_{i}$, namely: $X_{(1)}<X_{(2)}<\ldots<X_{(n)}$. This defines the color edge detection operator as follows:

$$
V R=\left\|X_{n}-X_{1}\right\|
$$

So that you can get a color image edge. This method can determine the color of the edge of the image, but it is likely to be noise to $X_{(n)}$,Therefore, this detection operator antinoise performance is relatively poor. On this basis, we get the minimum vector dispersion (MVD) edge detection operator by improve the color edge detection operators. Defined as follows:

$$
M V D=\min _{j}\left(\left\|X^{(n-j+1)}-\sum_{i=1}^{l} \frac{X^{(i)}}{l}\right\|\right)
$$

2) SIFT feature vector generation. SIFT algorithm take an eigenvalue test for image scale space to determine the location of key features and dimensions, and then use the direction key features and scale features to generate a feature vector unaffected from image scaling, rotation and brightness.

Such that the SIFT feature vector with scale invariant properties, SIFT algorithm take feature detection in scale space first. Scale space theory first appeared in the field of computer vision to simulate multi-scale features of image data. Existing literature prove Gaussian convolution kernel is to achieve a unique linear scaling transform kernel. Twodimensional Gaussian function is defined as follows:

$$
G(x, y, \sigma)=\frac{1}{2 \pi \sigma^{2}} e^{-\left(x^{2}+y^{2}\right) / 2 \sigma^{2}}
$$

The $\sigma$ is the variance of the Gaussian distribution. The two-dimensional images in different scales scale space is expressed as

$$
L(x, y, \sigma)=G(x, y, \sigma) * I(x, y)
$$

Wherein the symbol * represents the convolution operation, (x, y) represents the pixel position of the image, called $\sigma$ as scale space factor, the smaller the value is, the smoother the degree of the image, the corresponding scale is smaller. Large-scale overview of features representing the image, a small-scale representation of image detail features. $L$ represents the image scale space.

David G. Lowe detected local extreme points which exist in DoG scale space of the image and two-dimensional flat space as feature points [5], so that the image features have good independence and accuracy. DoG operator is defined through two different scales differential Gaussian kernel, and similar with LoG (Laplacian-of-Gaussian) operator. The DoG operators are as follows:

$$
\begin{aligned}
D(x, y, \sigma) & =(G(x, y, k \sigma)-G(x, y, \sigma)) * I(x, y) \\
& =L(x, y, k \sigma)-L(x, y, \sigma)
\end{aligned}
$$

For this obtained feature points, calculated the DoG response value in the scale space, and use these connecting values to obtain the scale of the feature point trajectory curve. Characteristic curve characteristic scales scale through a local extreme point to determine. Trajectory curve may have more than one local extreme point, that is, the feature points contained many characteristic scales.

SIFT feature vector generation algorithm includes four steps [6]:

a) the detection of scale space extremum;

b) pinpoint critical point;

c) determine the main direction of the key points;

d) SIFT feature vectors generated.

3) recursive partitioning feature point set. In order to cover the SIFT algorithm on the image, it need to obtain multiple images by geometric division, or get sets of feature point set by feature point division, for different image block or feature point set to be matched[7].

4) Match the SIFT feature vector. After obtained two SIFT feature point sets, the next step of feature vectors match can be divided into two steps:

a) similarity measure. Generally, people use the feature vector distance function as two key points in the image similarity measure. This paper uses Euclidean distance between two images as a similarity measure, which is 128-dimensional vector space distance between two points. After get the SIFT feature vectors, there using the priority k-d tree search to find the feature points of the two key points approximate nearest neighbor. In this two key points, if the nearest Euclidean distance divided by the next near Euclidean distance is less than a certain percentage threshold, this pair of matching points can be accept. With reduce this threshold, while lowering the SIFT matching points, but will make the detection more stable.

b) eliminate the mismatch. The potential matching pair can be acquire by the similarity metric analysis, in order to avoid some error in this matching process, so the constraints to elimination the errors in the matching process is indispensable, to improved robustness.

\section{The experimental results}

Experiments tested the CV-SIFT method with several typical digital images processing operation, including 
translation, rotation, scaling, brightness adjustment and combinations thereof, and matching points with the connection between these points which can be detected.

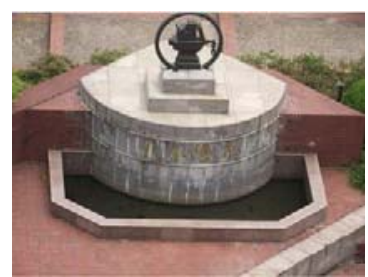

Figure 2. Artwork

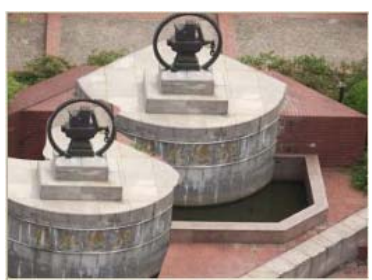

Figure 3. Tampering Figure

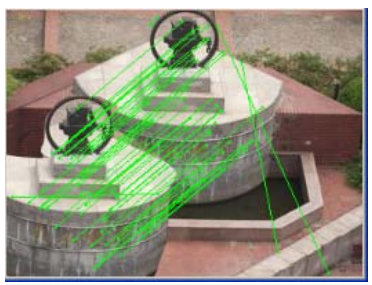

Figure 4. Result

In order to prove the accuracy of image detection with this algorithm, we collected 200 pictures, of which 100 is not a credible picture of tampering, including figures, landscapes, etc.; another 100 covered by the above pictures to get through replication, and covering all of these tampering operation and combinations thereof, as tampering pictures sets. The accuracy rate of this algorithm of detection shown in Table 1. Compare CV-SFIT algorithm with literature [8], the CV-SFIT algorithm supports detection for color images, and the accuracy rate is also improved. This algorithm accuracy rate near to $85.5 \%$.

TABLE I. DETECTION ACCURACY

\begin{tabular}{|l|c|c|}
\hline \multirow{2}{*}{} & \multicolumn{2}{|c|}{ Result } \\
\cline { 2 - 3 } & Natural & $\begin{array}{l}\text { Tamper } \\
\text { images }\end{array}$ \\
\hline $\begin{array}{l}\text { Not tampered } \\
\text { image library }\end{array}$ & $82 \%$ & $18 \%$ \\
\hline $\begin{array}{l}\text { Tampered } \\
\text { image library }\end{array}$ & $14.5 \%$ & $85.5 \%$ \\
\hline
\end{tabular}

\section{ACKNOWLEDGMENT}

This work was financially supported by the basic science project of Ministry of public security, project number: 2012GABJC035 and National Development and Reform commission, project number: [2012]1424.

\section{REFERENCES}

[1] Tomás Pevný, Jessica J. Fridrich, Andrew D. Ker: From Blind to Quantitative Steganalysis[J]. IEEE Transactions on Information Forensics and Security 7(2): 445-454 (2012)

[2] Hany Farid: Creating and Detecting Doctored and Virtual Images:Im Plieationsto the Child Pomorgaphy Prevention Act.Technical Report,Department of Computer Seience[J]. Dartmouht College, NewHampshire,USA ,2004

[3] Herbert Bay,Andreas Ess,Tinne Tuytelaars,Luc Van Gool”SURF:Speed Up Robust Features”,Computer Vision and Image Understanding(CVIU),Vol.110,No.3,pp.346-359,2008

[4] Rui Geng:An Image edge detection algorithm based on vector order statisticsComputer Engineering and Technology (ICCET), 2010 (2 ) 689-691

[5] LOWED G. Distinctive image features from scaleinvariant key points[J]. International Journal of Computer Vision, 2004, 60(2):91110

[6] Hany Farid: Exopsing Digital Forgeries in Scientific Images[A]. International MuItilmedia Conference Proceeding of the 8th workshop on Multimedia and security[C]. New York,NY,USA:ACM Press, 2006. 29-36.

[7] A C Popescu, H Farid: Exposing digital forgeries by detecting traces of re-sampling[J].IEEE Transactions on Signal Processing, 2005, v01. 53, no.2:758-767

[8] Qadir, Ghulam ; Xi Zhao ; Ho, Anthony T S ; Casey, M:Image forensic of glare feature for improving image retrieval using Benford'sLaw.Circuits and Systems (ISCAS), 2011 IEEE International Symposium o, 2011(1): 2661 - 2664 